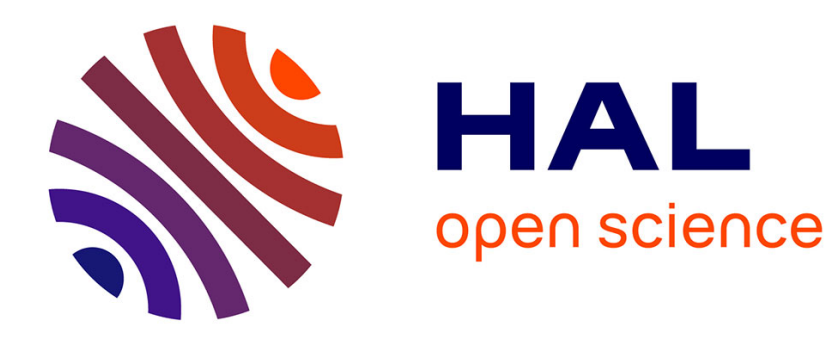

\title{
L'auto-soudage des tranches de silicium
}

F. Miserey

\section{To cite this version:}

F. Miserey. L'auto-soudage des tranches de silicium. Revue de Physique Appliquée, 1990, 25 (7), pp.763-773. 10.1051/rphysap:01990002507076300 . jpa-00246237

\section{HAL Id: jpa-00246237 https://hal.science/jpa-00246237}

Submitted on 1 Jan 1990

HAL is a multi-disciplinary open access archive for the deposit and dissemination of scientific research documents, whether they are published or not. The documents may come from teaching and research institutions in France or abroad, or from public or private research centers.
L'archive ouverte pluridisciplinaire HAL, est destinée au dépôt et à la diffusion de documents scientifiques de niveau recherche, publiés ou non, émanant des établissements d'enseignement et de recherche français ou étrangers, des laboratoires publics ou privés. 


\title{
Classification
}

Physics Abstracts

$68.48-61.60 \mathrm{C}-81.70 \mathrm{C}-82.65$

\section{L'auto-soudage des tranches de silicium}

\author{
F. Miserey \\ Conservatoire National des Arts-et-Métiers, Laboratoire de Physique des Composants Electroniques \\ (URA 827), 292 rue Saint-Martin, 75141 Paris Cedex 03, France \\ (Reçu le 12 férrier 1990, accepté le 2 avril 1990)
}

\begin{abstract}
Résumé. - On décrit une expérience dans laquelle des tranches de silicium de diamètre $10 \mathrm{~cm}$ ont été autosoudées. Les interfaces ont été caractérisées au moyen de plusieurs tests différents: l'un destructif (mécanique), les autres non destructifs (optique, par imagerie dans l'infra-rouge, et acoustique); des diodes PN tests ont été réalisées, également dans le but d'obtenir une cartographie des décohésions. L'aire effectivement soudée est supérieure (ou au moins égale) à $80 \%$ de la surface d'une tranche. On a étudié de manière quantitative les variations des dimensions des zones défectueuses en fonction de la température : on montre que les décohésions qui existent sur une même interface sont de deux types, selon que leurs dimensions augmentent ou, au contraire, diminuent lors d'un traitement thermique à $1200^{\circ} \mathrm{C}$.
\end{abstract}

\begin{abstract}
We report a self-bonding experiment with $10 \mathrm{~cm}$ diameter silicon wafers. Different methods were used to characterize the quality of the bonded interfaces : mechanical destructive testing, optical (by infrared imaging) and acoustical non-destructive testings ; PN test-diodes were used also to map the bonding voids. Bonded interfaces were found void-free over at least $80 \%$ of the wafer surface. Size variations of unbonded areas with temperature were quantitatively investigated : we show that bonding voids at an interface are of two kinds, according as their size increases or decreases with thermal treatment at $1200^{\circ} \mathrm{C}$.
\end{abstract}

\section{Introduction.}

La fabrication des dispositifs pour l'électronique de puissance nécessite de disposer de couches épaisses de silicium de haute résistivité et d'excellente qualité cristalline, qui permettent d'obtenir une tenue en tension élevée. On forme habituellement de telles couches par épitaxie sur un substrat homogène : ce procédé est d'autant plus difficile à mettre en œuvre que l'on recherche des épaisseurs fortes et des résistivités élevées. En pratique, l'épaisseur maximale que peut atteindre une couche épitaxiée n'excède guère la centaine de micromètres, et sa qualité cristalline se détériore progressivement pendant la croissance.

Une autre approche consiste à souder directement, c'est-à-dire sans apport de matière, deux tranches monocristallines ayant des résistivités différentes, la soudure devant obligatoirement être réalisée à une température inférieure à la température de fusion du silicium [1-3]. Ce soudage direct des plaquettes de silicium (ou auto-soudage du silicium) commence à être utilisé comme une alternative à l'épitaxie épaisse [4, 5].

Une variante consiste à oxyder les tranches de silicium avant de réaliser l'auto-soudure, de manière à obtenir une structure $\mathrm{Si} /$ oxyde/Si, qui constitue un point de départ intéressant pour la fabrication de certains composants et de capteurs [6-12].

Il peut arriver que les tranches de silicium ne surface : des décohésions existent alors à l'interface, correspondant aux zones où la liaison directe $\mathrm{Si}-\mathrm{Si}$ n'a pu s'établir entre les deux surfaces. Ces décohésions peuvent avoir différentes origines :

(a) la présence de corps étrangers (particules, espèces chimiques amenées ou libérées par les traitements subis);

(b) une planéité insuffisamment bonne à l'échelle des mailles cristallines, résultant :

- de dommages physiques (micro-dépressions, par exemple) créés lors du découpage des tranches, 
et que le polissage des surfaces ne permet pas toujours de réparer,

- de l'existence de contraintes (ordre de grandeur : $10^{6}$ Pascal), certaines (internes au silicium) étant libérées par le traitement thermique, d'autres pouvant naître de l'alignement, nécessairement imparfait, des réseaux cristallins des deux tranches,

- d'un état de surface caractérisé par une rugosité superficielle de trop grande amplitude moyenne (supérieure à la dizaine de nanomètres).

2. Mise en æuvre d'un procédé d'auto-soudage pour des tranches de diamètre $10 \mathrm{~cm}$; étude qualitative des interfaces $\mathrm{Si} / \mathrm{Si}$ obtenues.

2.1 MOdE OPÉRATOIRE. - Des tranches de silicium monocristallin de diamètre $100 \pm 0,5 \mathrm{~mm}$, et d'épaisseur $550 \pm 25 \mu \mathrm{m}$ ont été utilisées. Elles possèdent toutes au moins une face polie de façon mécanicochimique, la mise en contact de deux tranches devant s'effectuer par les faces polies. Il s'agit de tranches commercialement disponibles, dont la qualité et les spécifications vérifient des normes courantes. En particulier, leur planéité n'est pas garantie à mieux de $5 \mu \mathrm{m}$ (soit environ $10^{4}$ fois la maille cristalline).

A la différence d'autres techniques de soudage (celle du «soudage assisté par champ électrique »[13-15], par exemple), la technique d'autosoudage du silicium ne nécessite pas d'appliquer une force extérieure.

Les tranches subissent un nettoyage poussé en salle blanche de classe $<100$. Leur soudage est réalisé en trois étapes successives : traitement chimique, mise en contact et adhésion à la température ordinaire, traitement thermique.

2.1.1 Traitement chimique. - Par oxydation en phase liquide, on forme à la surface des tranches une mince couche d'oxyde $\mathrm{SiO}_{x}$ [16]. La composition du bain acide utilisé et sa température, ainsi que la durée du traitement, permettent d'estimer à quelques nanomètres l'épaisseur de la couche d'oxyde formée [17]. Les tranches sont ensuite baignées dans l'eau courante désionisée, puis séchées à l'air chaud filtré. $\mathrm{Au}$ terme de ce traitement chimique, les surfaces doivent être recouvertes par des monosilanols $\mathrm{Si}-\mathrm{OH}[18,19]$.

2.1.2 Adhésion. - Les tranches sont mises en contact (à la température ordinaire) et adhèrent spontanément l'une à l'autre.

Une interaction moléculaire attractive se développant de plaquette à plaquette entre espèces chimiques (groupes $\mathrm{OH}$ ) doit être directement et complètement responsable de l'adhérence des plaquettes [20]. Suivant l'hypothèse de Lasky [21], cette interaction ne serait autre que la liaison hydrogène $[22,23]$.
L'adhésion se produit initialement dans une zone où le contact est plus étroit qu'ailleurs, et se propage ensuite (onde d'adhésion) avec l'extension de cette zone de contact étroit à toute la surface [24].

L'opération de mise en contact s'effectue en utilisant un support spécialement conçu pour permettre la superposition la plus exacte possible de deux tranches, face polie contre face polie, en tirant parti de l'existence du méplat pour respecter au mieux leur orientation cristalline.

2.1.3 Traitement thermique. - Les paires de tranches auto-adhérentes sont ensuite portées à $T=180^{\circ} \mathrm{C}$ pendant 3 heures sous flux d'azote.

Pour $T$ augmentant jusqu'à $500^{\circ} \mathrm{C}$, une réaction de déshydratation-condensation remplace certainement les liaisons entre silanols $(\mathrm{SiOH})$ par des ponts siloxanes ( $\mathrm{SI}-\mathrm{O}-\mathrm{Si}$ ) entre les 2 plaquettes, suivant [25] :

$$
\mathrm{Si}-\mathrm{OH}+\mathrm{OH}-\mathrm{Si} \rightarrow \mathrm{Si}-\mathrm{O}-\mathrm{Si}+\mathrm{H}_{2} \mathrm{O} .
$$

Lorsque $T$ dépasse $1000^{\circ} \mathrm{C}$ (mais reste très inférieure à la température de fusion du silicium : $1400^{\circ} \mathrm{C}$ ), on pense que les atomes d'oxygène cessent de servir d'intermédiaires entre les atomes de silicium des 2 surfaces, ces derniers se réarrangant pour former des liaisons directes $\mathrm{Si}-\mathrm{Si}$.

\subsection{ETUDE QUALITATIVE DES PAIRES DE TRANCHES AUTO-SOUDÉES.}

2.2.1 Test destructif. - La paire de tranches soudées est fracturée en suivant des lignes préalablement tracées à l'aide d'un diamant sur l'une des tranches (Fig. 1). La paire est d'abord coupée en deux, puis chaque morceau est à nouveau partagé, et ainsi de suite. On obtient finalement des morceaux dont les dimensions sont approximativement $5 \times 5 \mathrm{~mm}$. Dans les zones non ou partiellement soudées, la cassure s'effectue en s'écartant du tracé et s'accompagne de l'écaillage de l'une des tranches de silicium; alors que dans les zones bien soudées la cassure est à bords francs, les deux tranches restant parfaitement solidaires l'une de l'autre. L'aire effectivement soudée a été évaluée au moyen de ce test :

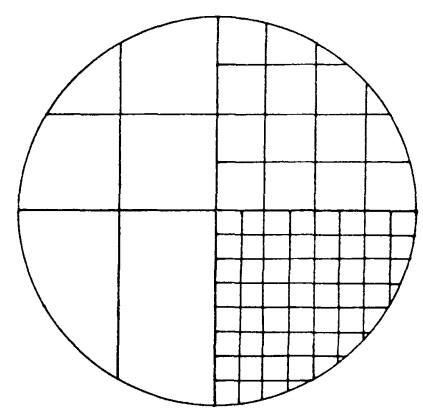

Fig. 1. - Test mécanique destructif.

[Cutting test.] 
elle atteint $80 \%$ de l'aire totale des surfaces assemblées.

2.2.2 Tests non destructifs. - L'emploi d'ondes infrarouges ou ultrasonores pour lesquelles le silicium est transparent permet de localiser les décohésions à l'interface. Nous utilisons une caméra infrarouge (Inframetrics $610 \mathrm{~A}$ ) dont les détecteurs, refroidis à $77 \mathrm{~K}$, sont sensibles respectivement dans les bandes de longueurs d'onde 3-5 et 8-12 $\mu \mathrm{m}$. L'optique de la caméra conjugue l'interface de soudure avec la surface sensible du détecteur (cf.

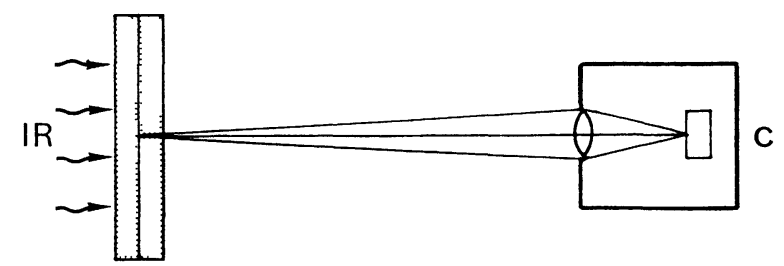

Fig. 2. - Utilisation d'une caméra infrarouge (C) pour localiser les défauts de soudure.

[Mapping the bonding voids with the help of an infrared camera (C).]
Fig. 2). On obtient une image de l'interface lorsque celle-ci est éclairée à travers l'une des deux tranches par un rayonnement infra-rouge de spectre étendu.

La paire soudée constitue une lame à faces parallèles, d'épaisseur $2 e$. Là où les tranches ne sont pas en contact étroit, existe une petite lame d'épaisseur $\varepsilon \ll e$, emplie - ou non - de gaz, ce qui affecte le déphasage entre deux faisceaux transmis successifs. On obtient ainsi l'image d'un défaut à l'interface par contraste interférentiel.

Un cas important à considérer est celui où l'épaisseur $\varepsilon$ ne serait pas uniforme et prendrait des valeurs ayant l'ordre de grandeur des longueurs d'onde des radiations infra-rouges utilisées : on pourrait alors observer des franges d'égale épaisseur, pratiquement localisées sur la plaque de silicium, et ceci bien que la source soit étendue et la lumière polychromatique.

La luminance $L$ des images fournies par la caméra est mesurée à l'aide d'une unité arbitraire. Les différentes images obtenues sont comparables entre elles, la température et l'émissivité de la source restant constantes.

Une image typique de celles que nous pouvons ainsi obtenir est montrée sur la figure 3 . Les trois

\section{Infmentos}

\section{Therotalnix}

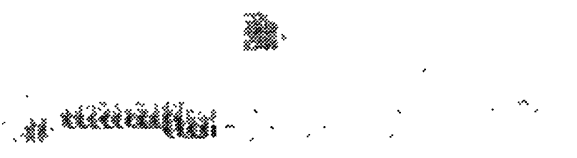

Fig. 3. - Image infrarouge d'une paire de tranches $\mathrm{Si} / \mathrm{Si}$ auto-soudées. Les zones sombres correspondent à des défauts de soudure.

[Infrared image of a self-bonded $\mathrm{Si} / \mathrm{Si}$ wafer pair. Uncontacted areas are dark.] 
zones où la luminance diffère de celle du "fond » correspondent effectivement à des défauts de soudure à l'interface, comme cela a été vérifié en fin d'expérience à l'aide du test mécanique destructif. Le nombre de défauts observés (3), ainsi que la surface qu'ils occupent (quelques $\mathrm{cm}^{2}$ ), sont représentatifs de l'état actuel de notre savoir-faire en matière d'auto-soudage.

La figure 4 montre des images, obtenues par trois méthodes distinctes, qui correspondent à une même paire de tranches auto-soudées (choisie dans un souci pédagogique en raison du nombre non négligeable de défauts qu'elle possède).

- Sur l'image (4a) obtenue à la caméra infrarouge dans la bande $3-5 \mu \mathrm{m}$, on observe huit défauts (repérés par les lettres A à $\mathrm{H}$ ), qui occupent des surfaces d'aires nettement différentes, comprises entre $1 \mathrm{~mm}^{2}$ et quelques $\mathrm{cm}^{2}$; leur contraste $\gamma$, défini par $\gamma=\left(L-L^{\prime}\right) / L$ (où $L$ est la luminance du "fond", et $L^{\prime}$ celle de la partie centrale de l'image du défaut), varie entre $0,5 \%$ et $7 \%$.

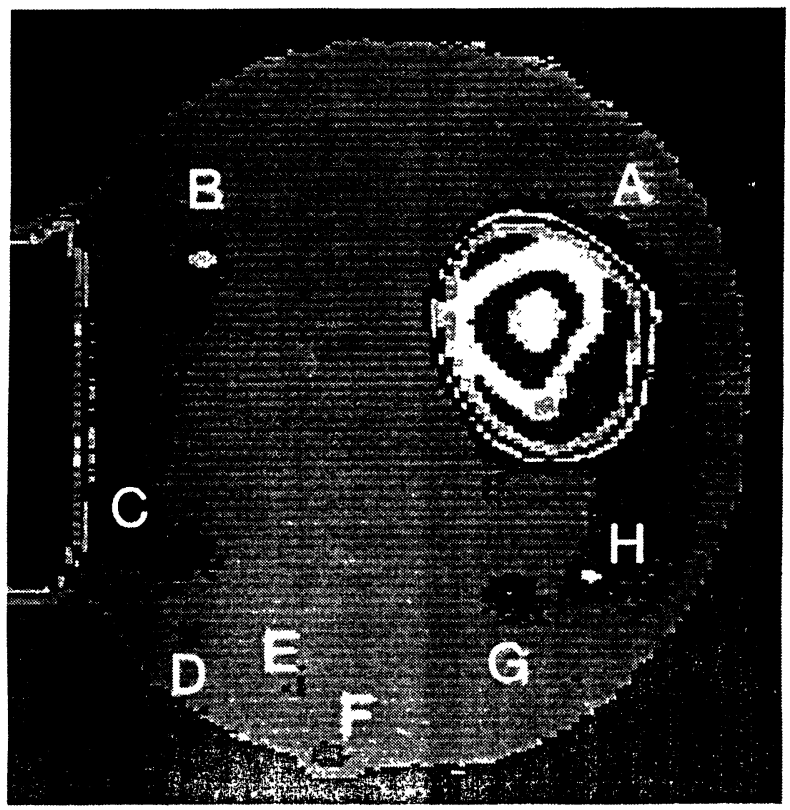

a)

Fig. 4. - a) Image infrarouge $(3<\lambda<5 \mu \mathrm{m})$ de l'interface entre deux tranches de silicium auto-soudées présentant au moins huit décohésions (A-H). b) Image en réflexion à $\lambda=1,5 \mu \mathrm{m}$, obtenue par balayage, de la même interface qu'à la figure $6 \mathrm{a}$. c) Image acoustique à la fréquence $25 \mathrm{MHz}$ de la même paire de tranches qu'à la figure $6 \mathrm{a}$.

[a) Infrared $(3<\lambda<5 \mu \mathrm{m})$ image of the interface between two self-bonded silicon wafers : some eight voids (A-H) are observed. b) Reflection image $(\lambda=1,5 \mu \mathrm{m})$, of figure $6 \mathrm{a}$ interface, obtained by scanning. c) Acoustograph $(f=25 \mathrm{MHz})$ of figure $6 \mathrm{a}$ wafer pair.]
- L'image (4b) a été fournie par un système automatisé d'imagerie par balayage, construit au CNAM, et dont le schéma de principe est représenté sur la figure 5 : un faisceau infra-rouge approximativement monochromatique $(\lambda=1,5 \mu \mathrm{m})$ éclaire sous l'incidence $45^{\circ}$ la paire de tranches soudées; le faisceau réfléchi est reçu par une photodiode au germanium; une table $\mathrm{X}-\mathrm{Y}$ motorisée permet de déplacer la paire de tranches, de manière à ce qu'elle soit explorée point par point par le faisceau infrarouge, avec un pas de $1 \mathrm{~mm}$. Dans cette méthode d'examen par réflexion, l'existence d'un défaut se traduit par un maximum de signal. On retrouve les huit défauts observés en (4a), mais avec un plus grand contraste.

- L'image (4c) a été obtenue au microscope acoustique (Honda HA 711) en utilisant des ondes longitudinales ultrasonores de fréquence $25 \mathrm{MHz}$. Lorsqu'une différence d'impédance acoustique existe, due à une décohésion à l'interface entre les deux tranches de silicium, la réflexion des ondes

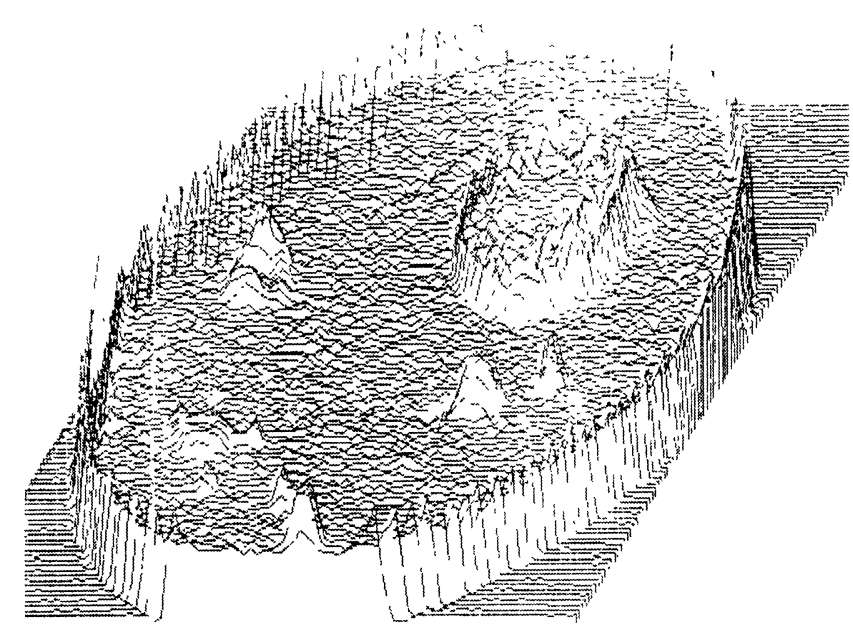

b)

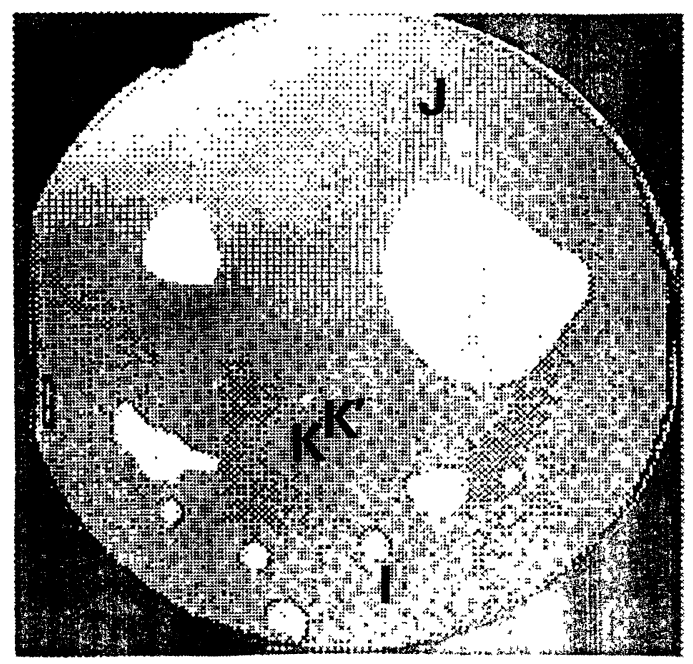

c) 


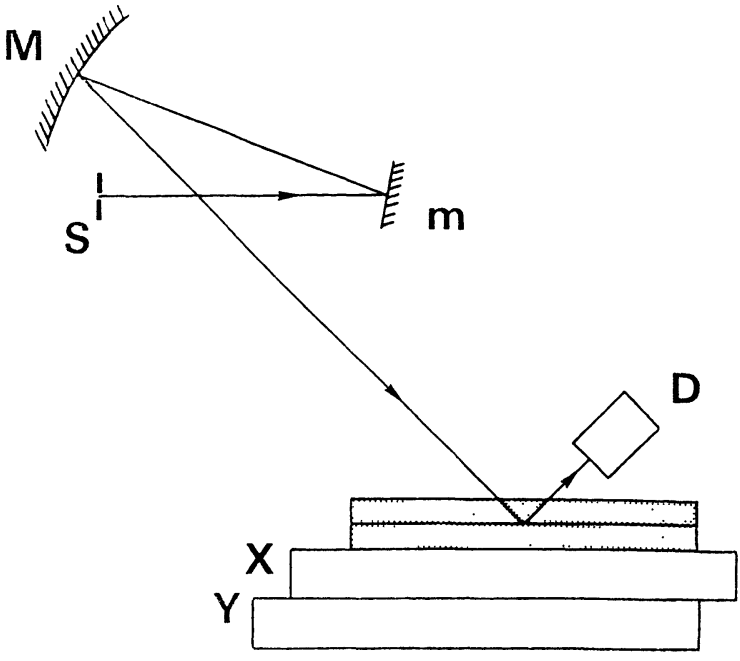

Fig. 5. - Schéma du dispositif d'imagerie infrarouge à balayage. $\mathrm{S}$ : source monochromatique $(\lambda=1,5 \mu \mathrm{m})$; $\mathrm{m}$ : miroir plan; $\mathbf{M}$ : miroir concave; $\mathrm{D}$ : diode au germanium; $\mathrm{X}$ et $\mathrm{Y}$ : tables de déplacement.

[Experimental set-up diagram of the infrared scanning imager. $S$ : monochromatic light source $(\lambda=1,5 \mu \mathrm{m})$; $\mathrm{m}$ : plane mirror; $\mathbf{M}$ : concave mirror; $\mathbf{D}$ : germanium diode $; \mathrm{X}$ and $\mathrm{Y}$ : translation tables.]

donne naissance aux taches claires observées sur l'image. La luminance du "fond » de l'image n'est pas uniforme : ceci est dû à la non-planéité ("warpage") de la lame formée par la paire de tranches, qui affecte sensiblement la réflexion des ondes acoustiques sur la face d'entrée. On retrouve, mais avec une meilleure définition, tous les défauts déjà observés par les techniques en infra-rouge ; quatre défauts: $\mathbf{I}, \mathrm{J}, \mathrm{K}$ et $\mathrm{K}^{\prime}$, les deux derniers occupant une surface relativement faible $\left(s<1 \mathrm{~mm}^{2}\right)$, sont de plus observés. Par contre, cette image acoustique, telle qu'elle est, ne fournit pas d'information sur l'amplitude des décohésions : les taches correspondantes ont toutes approximativement la même luminance.

2.2.3 Diodes tests. - Dans le but de dresser une cartographie électrique de deux tranches de silicium auto-soudées, on a réalisé des diodes PN tests. Une

polies a été auto-soudée à une tranche $\mathrm{N}^{+}$d'épaisseur $550 \mu \mathrm{m}$ épitaxiée $\mathrm{N}$ dont une seule face était polie. Une région $\mathrm{P}$ a été ensuite formée au moyen d'un dépôt homogène de Bore sur la face avant $\left(\mathrm{N}^{-}\right)$de la paire soudée. Après métallisation, cette face a été sillonnée au pas de $1 \mathrm{~mm}$, avec une profondeur approximative de $20 \mu \mathrm{m}$ (cf. Fig. 6). Près de 6000 diodes rudimentaires ont été ainsi réalisées. De manière à mettre en évidence d'éventuelles variations de la résistance série des diodes, leur chute de tension directe a été mesurée pour un

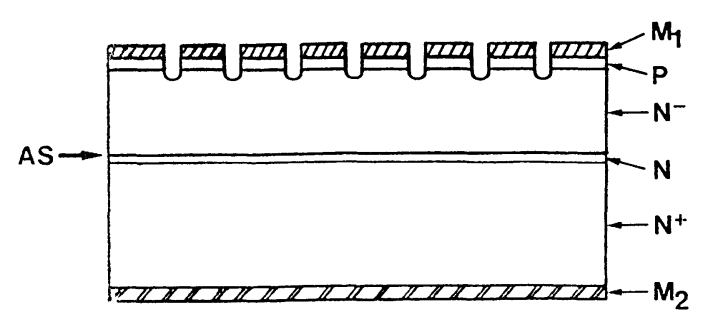

Fig. 6. - Diodes PN tests $\left(M_{1}\right.$ et $M_{2}$ : dépôts métalliques ; $\mathrm{P}, \mathbf{N}^{-}, \mathbf{N}, \mathbf{N}^{+}$: couches et substrats de différents dopages ; AS : auto-soudure).

[PN testing diodes $\left(M_{1}\right.$ and $M_{2}:$ metallizations $; \mathrm{P}, \mathrm{N}^{-}, \mathrm{N}$, $\mathrm{N}^{+}$: doped layers and bulks; AS : self-bonding interface.]

courant direct d'intensité constante, et à raison d'une diode pour 25 réalisées. Sur 237 diodes testées, 190 (c'est-à-dire $80 \%$ d'entre elles) ont une tension directe comprise entre 1 et 1,5 volt lorsqu'elles sont traversées par un courant direct d'intensité $I=50 \mathrm{~mA}$. On a coché sur la figure 7 a les zones de $5 \times 5 \mathrm{~mm}$ correspondant à une diode testée pour laquelle la tension directe était en-dehors de ces limites.

Après avoir ôté les métallisations des deux faces, on a examiné la plaque de diodes en lumière infrarouge. La figure $7 \mathrm{~b}$ montre l'image obtenue par réflexion dans la bande 3-5 $\mu \mathrm{m}$ à l'aide de la caméra infra-rouge. Les défauts localisés par ce moyen correspondent aux zones repérées comme étant " hors limites» sur la figure 7a. Cette correspondance s'interprète aisément en considérant que les lignes de courant traversant la plaquette $\mathbf{N}^{-}$doivent contourner les décohésions existant à l'interface, ce qui accroît localement la résistance série des diodes.

(Le nombre relativement élevé de défauts de soudure observés est imputable à la qualité médiocre du substrat $\mathrm{N}^{+}$épitaxié $\mathbf{N}$ utilisé dans cette expérience, une inspection optique de ce lot de tranches ayant révélé la présence d'une densité anormalement élevée de pics d'épitaxie à la surface polie de ces tranches.)

\section{Etude quantitative : effet du traitement thermique à $1200^{\circ} \mathrm{C}$ sur la dimension des aires non soudées.}

Plusieurs auteurs se sont intéressés à l'effet du traitement thermique sur les décohésions, en relation avec l'ambiance gazeuse dans laquelle les plaquettes sont mises en contact: le caractère contradictoire des résultats publiés à ce jour nous a amenés à réaliser la présente expérience.

Des tranches de silicium (100), les unes du type $\mathrm{N}^{+}$(résistivité $30 \mathrm{~m} \Omega . \mathrm{cm}$ ) les autres du type $\mathrm{N}$ (résistivité $40 \Omega . \mathrm{cm}$ ) sont traitées chimiquement suivant le mode opératoire décrit en 2.1 et assemblées de manière à former des paires $\mathrm{N}^{+} \mathrm{N}$. Leur mise en 


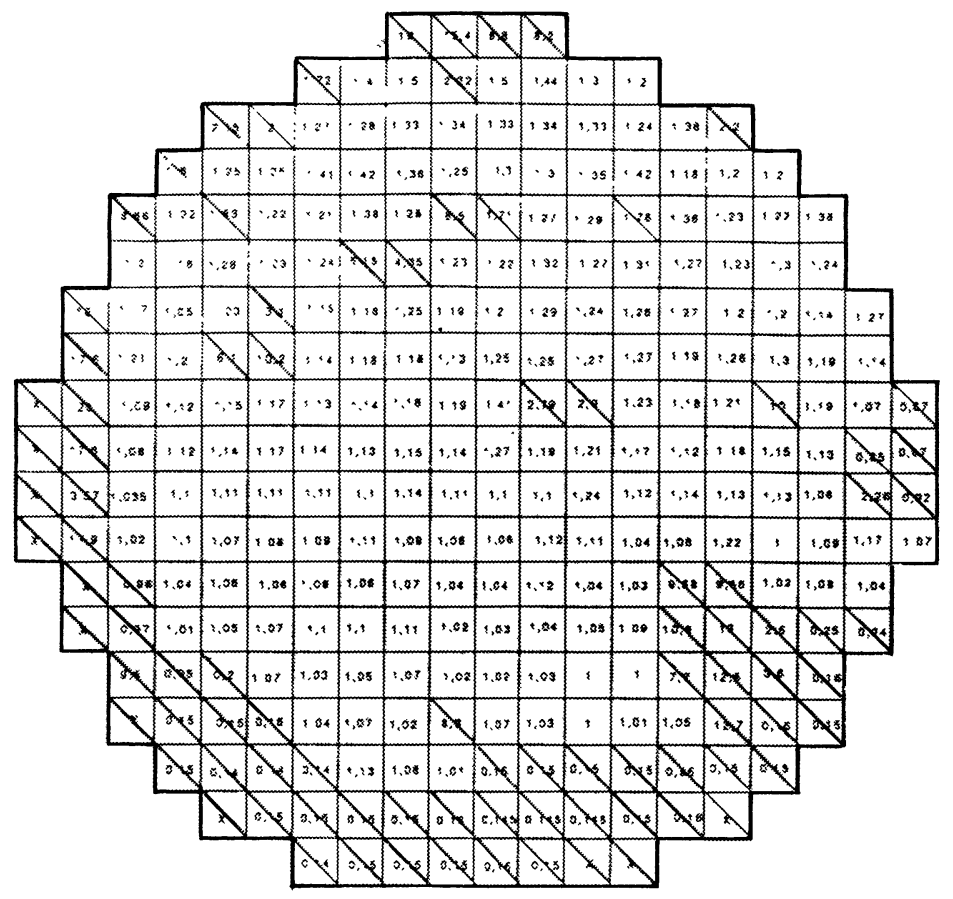

(a)

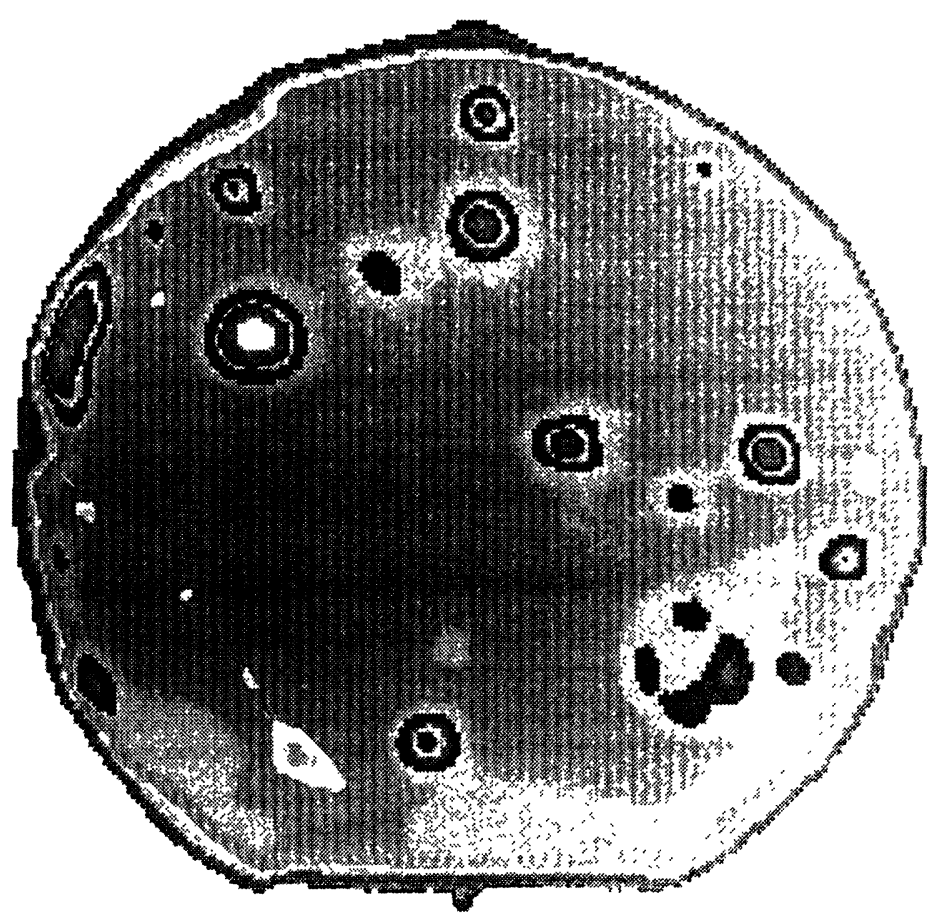

(b)

Fig. 7. - Paire de tranches auto-soudées utilisée pour réaliser des diodes-tests. (a) Carte de la tension directe des diodes pour $I=50 \mathrm{~mA}$; les valeurs qui s'écartent notablement de la moyenne ont été cochées. (b) Image infrarouge (obtenue après avoir ôté les métallisations) montrant les zones non soudées.

[Processed wafer pair. (a) Map of diode forward voltage for $I=50 \mathrm{~mA}$; abnormal values are ticked off. (b) Infrared image (after the metallizations have been removed) showing the unbonded areas.] 
contact s'effectue soit à l'air (cas de la paire I) soit sous flux d'oxygène (cas de la paire II).

Une première image des deux paires est faite à l'aide de la caméra infra-rouge, de manière à locali- ser et à caractériser les régions de l'interface où les surfaces ne sont pas intimement en contact à ce stade de l'expérience. Les paires subissent ensuite le traitement thermique. Une nouvelle image est faite,

(1)

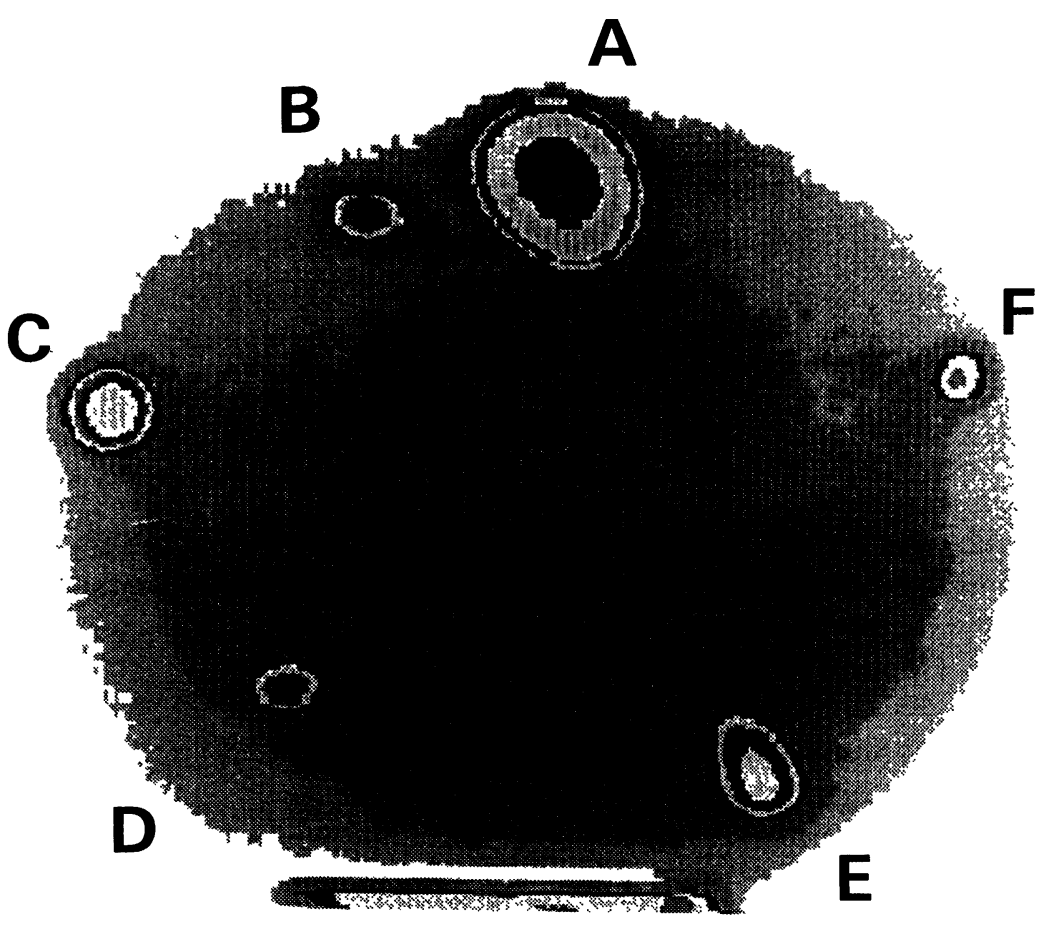

(2)

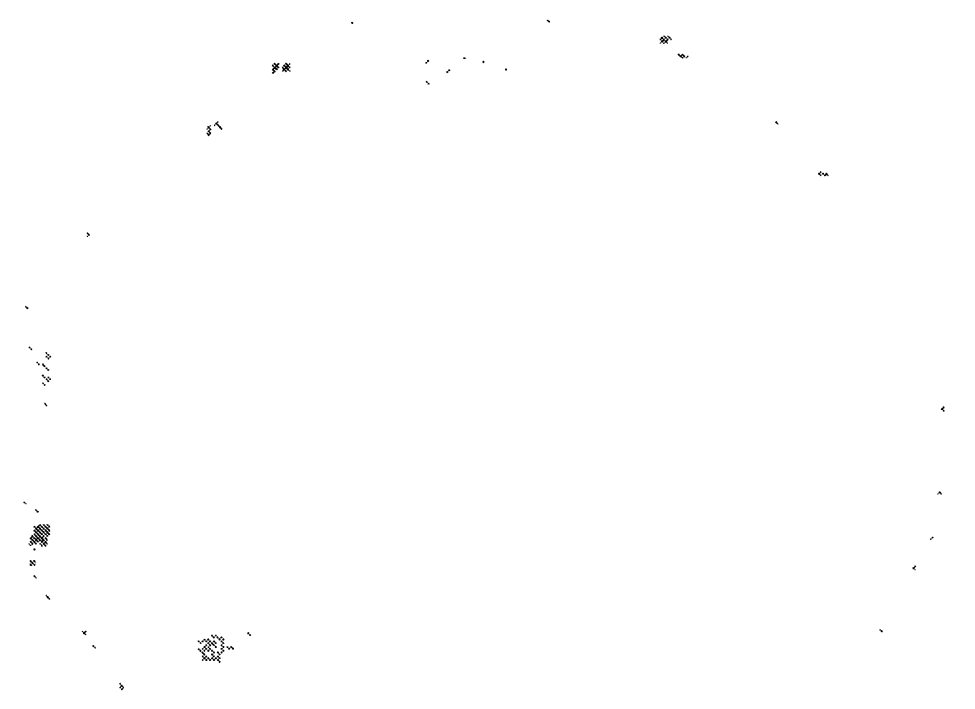

Fig. 8. - Image infrarouge de la paire $\mathrm{I}:$ (1) après la mise en contact des tranches dans l'air à $T=300 \mathrm{~K}$; (2) après le traitement thermique.

[Infrared image of wafer pair I : (1) after contacting the wafers in air at $T=300 \mathrm{~K}$; (2) after the thermal treatment.] 
après retour à la température ambiante ordinaire, dans le but de mettre en évidence les défauts de soudure.

Une source de chaleur régulée à température constante est placée à proximité et en vue directe de la tranche $\mathrm{N}^{+}$. Le dopage relativement grand de cette tranche la rend opaque au rayonnement IR dans les bandes où la caméra est sensible. Sa température s'élève légèrement au-dessus de l'ambiante, et le rayonnement qu'elle émet alors dans la bande 8-12 $\mu \mathrm{m}$ est vu par la caméra à travers l'interface $\mathrm{N}^{+} / \mathrm{N}^{-}$: ce rayonnement traverse la tranche $\mathrm{N}^{-}$qui est faiblement dopée et relativement bien transparente à ces longueurs d'onde. Les défauts sont dénombrés, leur aire $s$ mesurée, ainsi que leur contraste $\gamma$.

La figure 8 montre les images de la paire (I) : avant le traitement thermique, sept défauts sont visibles, dont l'aire est comprise entre 10 et $260 \mathrm{~mm}^{2}$, et dont le contraste n'excède pas $6 \%$ (cf. Tab. I); les sept défauts existent encore après le traitement thermique, mais on remarque que $s$ et $\gamma$ ont diminué pour quatre d'entre eux $(\mathrm{B}, \mathrm{C}, \mathrm{E}, \mathrm{F})$ et ont au contraire augmenté pour les trois autres (A, D, G).

La figure 9a montre, en prenant l'exemple du défaut $\mathrm{F}$, comment, en règle générale, la luminance $I^{\prime}$ d'un défaut décroît de façon monotone du bord vers le centre lorsqu'on se déplace suivant un diamètre. Il n'en va pas de même dans le cas particulier du défaut A (Fig. 9b) : on note, avant le traitement thermique, l'existence d'une frange
Tableau I. - Défauts de soudure de la paire (I) de tranches assemblées dans l'air : aire $s$, contraste $\gamma$, et leurs variations relatives avec le traitement thermique. [Unbonded areas in air-contacted wafer pair (I): area $s$, contrast $\gamma$, and their relative change with thermal treatment.]

\begin{tabular}{|c|c|c|c|c|}
\hline Défaut & $s(\mathrm{~mm})^{2}$ & $\gamma(\%)$ & $\frac{\Delta s}{s}(\%)$ & $\frac{\Delta \gamma}{\gamma}(\%)$ \\
\hline A & 260 & $(3,3)\left(^{*}\right)$ & +9 & $(+24,2)\left(^{*}\right)$ \\
\hline B & 56,5 & 4,1 & -29 & -39 \\
\hline C & 93,5 & 5,7 & $-3,5$ & -14 \\
\hline D & 41,5 & 3,3 & +41 & $+48,5$ \\
\hline E & 115 & 4,1 & -99 & $-79,2$ \\
\hline F & 41,5 & 3,3 & -46 & $-75,1$ \\
\hline G & 10,5 & 0,85 & $+28,5$ & +194 \\
\hline
\end{tabular}

(*) $\gamma$ désigne, dans ce cas particulier, le contraste entre la frange centrale et le « fond».

d'égale épaisseur (centre clair, entouré d'un anneau sombre); le traitement thermique modifie le nombre de franges, en faisant apparaître une demi-frange supplémentaire, ce qui indique que l'épaisseur du

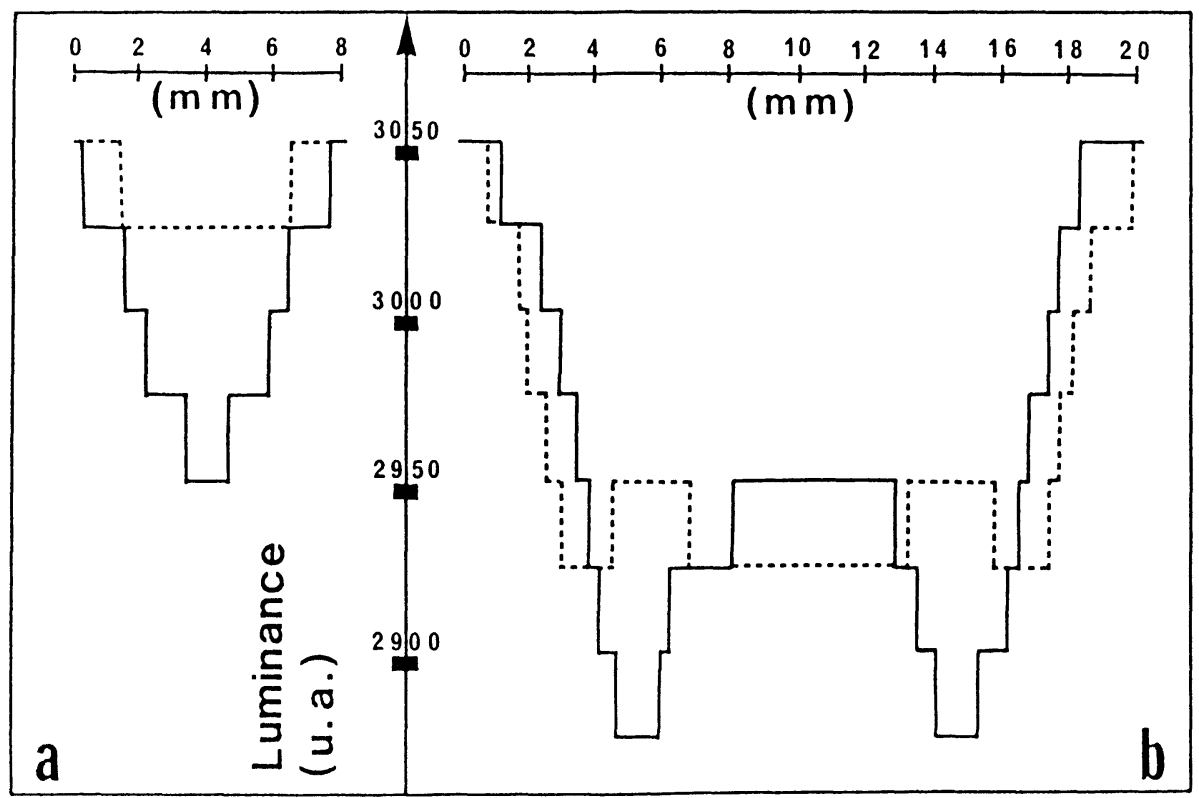

Fig. 9. - Variation de la luminance le long d'un diamètre: (a) du défaut F, (b) du défaut A, avant (en trait plein) et après (en trait interrompu) le traitement thermique \{ u.a. : unité arbitraire $\}$.

[Luminance variation along a diameter of : (a) defect F, (b) defect A, before (full line) and after (dashed line) the thermal treatment $\{$ u.a. : arbitrary unit $\}$.] 
défaut, initialement de l'ordre de $5 \mu \mathrm{m}$, a augmenté d'environ $50 \%$.

La figure 10 montre les images de la paire (II) : des sept défauts visibles avant le traitement thermi- que, quatre $(a, b, c, e)$ disparaissent après ce traitement, deux (d, g) diminuent d'aire et de contraste, un seul (f) augmente d'aire sans que le contraste change (cf. Tab. II).

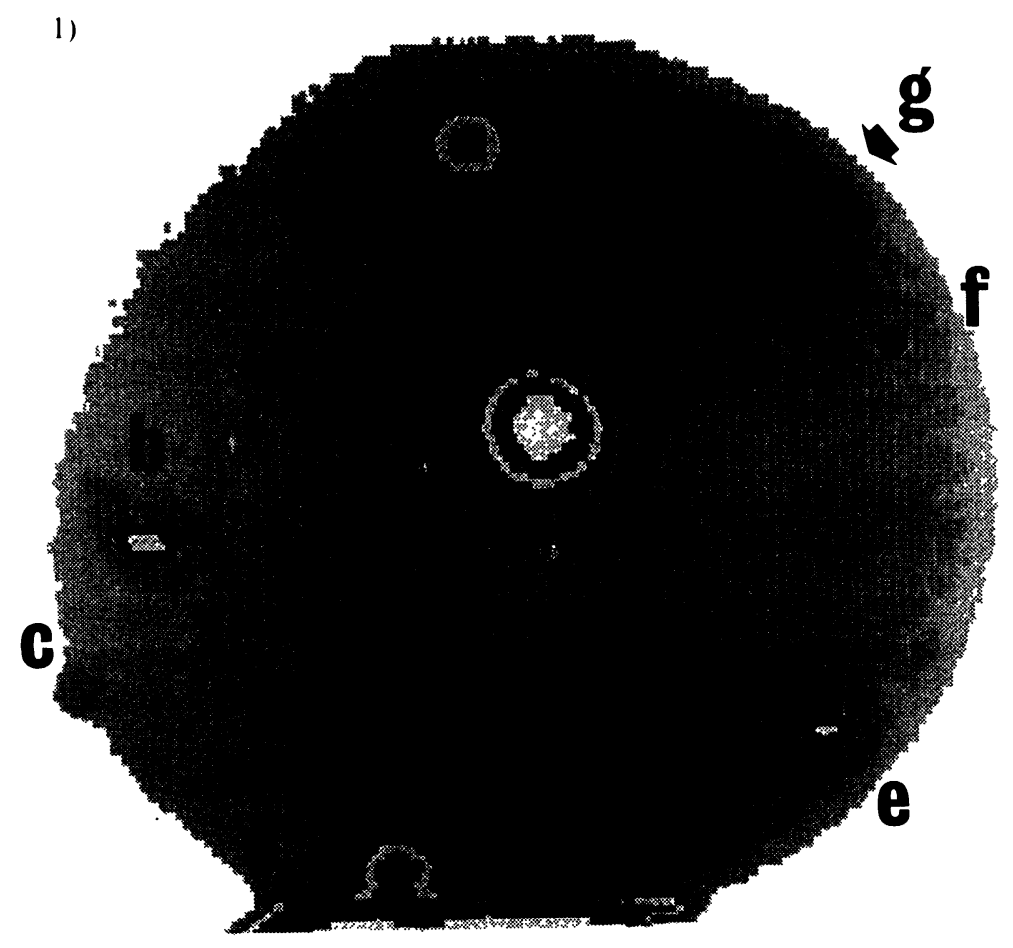

2)

Fig. 10. - Image infrarouge de la paire II : 1) Après la mise en contact des tranches sous flux d'oxygène à $T=300 \mathrm{~K}$; 2) Après le traitement thermique.

[Infrared image of wafer pair II : 1) After contacting the wafers under oxygen flow at $T=300 \mathrm{~K}$; 2) After the thermal treatment.] 
Tableau II. - Défauts de soudure de la paire (II) de tranches assemblées sous oxygène : aire s, contraste $\gamma$, et leurs variations relatives avec le traitement thermique.

[Unbonded areas in oxygen-contacted wafer pair (II) : area $s$, contrast $\gamma$, and their relative change with thermal treatment.]

\begin{tabular}{|c|c|c|c|c|}
\hline Défaut & $s\left(\mathrm{~mm}^{2}\right)$ & $\gamma(\%)$ & $\frac{\Delta s}{s}(\%)$ & $\frac{\Delta \gamma}{\gamma}(\%)$ \\
$\mathrm{a}$ & 48,5 & 2,6 & -100 & -100 \\
\hline $\mathrm{b}$ & 57,5 & 2,6 & -100 & -100 \\
\hline $\mathrm{c}$ & 10 & 0,86 & -100 & -100 \\
\hline $\mathrm{d}$ & 57,5 & 2,6 & $-23,5$ & $-66,9$ \\
\hline $\mathrm{e}$ & 80 & 1,74 & -100 & -100 \\
\hline $\mathrm{f}$ & 10 & 0,87 & $+132,5$ & 0 \\
\hline $\mathrm{g}$ & 133 & 4,28 & $-62,5$ & $-80,6$ \\
\hline
\end{tabular}

Pour tous les défauts autres que $\mathrm{A}$, on admet que le contraste $\gamma$ est proportionnel à l'épaisseur $\varepsilon \mathrm{du}$ défaut (ce qui n'est en toute rigueur valable que si le défaut est assimilable à une petite lame éclairée en lumière monochromatique par un faisceau parallèle).

Ceci étant admis, nos résultats montrent que, d'une part, les défauts de soudure sont de deux types, selon que leurs dimensions $(s, \varepsilon)$ augmentent ou, au contraire, diminuent sous l'effet du traitement thermique, et que, d'autre part, ces deux types de défauts coexistent sur un même échantillon. Nous observons que la diminution des dimensions peut aller jusqu'à entraîner la disparition d'un défaut à la suite du traitement thermique.

Un groupe d'expérimentateurs [1] [26] a observé que les défauts apparaissaient pendant le traitement thermique, pour $T>200^{\circ} \mathrm{C}$, mais qu'ils disparaissaient ensuite complètement pour $T>800^{\circ} \mathrm{C}$. Par contre, d'autres expérimentateurs $[27,28]$ observent des défauts dès la température ambiante ordinaire, et ces défauts ne seraient pas modifiés par le traitement thermique.

En comparant nos résultats obtenus avec les deux paires $\mathrm{N}^{+} / \mathrm{N}^{-}$, nous constatons que la qualité de la soudure directe est meilleure si l'opération de mise en contact des plaquettes est effectuée sous oxygène plutôt que dans l'air. Ce point a également été souligné par Black et al. [3].

Les deux types de défauts que nous avons distingués en fonction de leur évolution avec la température ont certainement des origines différentes : (a) l'augmentation des dimensions d'un défaut s'explique en supposant qu'une particule (éclat de silicium, par exemple) fait localement obstacle au rapprochement des plaquettes. La décohésion se comporterait alors comme un piège, où viendraient s'accumuler des atomes étrangers au monocristal; (b) il en va autrement si la décohésion est constituée par une micro-cuvette uniquement remplie de gaz emprisonné lors de la mise en contact des plaquettes. A haute température, le volume emprisonné peut diminuer par suite de la diffusion du gaz vers l'intérieur des plaquettes. D'autre part, l'augmentation de la plasticité du silicium à ces températures autorise la déformation du solide [24]. Cette explication rend compte des résultats plus favorables obtenus lorsque l'adhésion des plaquettes est réalisée sous flux d'oxygène, ce gaz diffusant relativement vite dans le silicium.

\section{Conclusion.}

Nous avons expérimenté un procédé permettant l'auto-soudage de tranches de silicium monocristallin (diamètre des tranches : $10 \mathrm{~cm}$ ). Des tests différents (mécanique, optique, acoustique, électrique) ont donné des résultats très concordants quant à l'existence et à la localisation, à l'interface entre deux tranches soudées, de zones non adhérentes (ou décohésions) occupant au plus $20 \%$ de la surface d'une tranche. L'étude quantitative de ces décohésions en lumière infra-rouge montre: (a) qu'elles sont de deux sortes, suivant leur comportement en température ; (b) qu'elles sont moins nombreuses et de plus faibles dimensions dans le cas où les tranches ont été mises en contact sous flux d'oxygène. Ces résultats ont été obtenus avec des tranches de silicium commercialement disponibles, de spécifications courantes, et en travaillant en salle blanche de classe inférieure à 100 . Un taux de défauts plus faible devrait pouvoir être obtenu au prix : (a) d'une sélection initiale des tranches; (b) de précautions supplémentaires qu'il faudrait prendre pour accroître la propreté de l'environnement et du mode opératoire de l'auto-soudage.

\section{Remerciements.}

Ce travail a été principalement effectué à la Direction Recherche et Développement de la Société Télémécanique à Nanterre, au sein du groupe "Recherche Technologies Semiconducteurs". L'auteur remercie tous les membres de l'équipe «Silicium » pour leur excellent accueil et leur constante coopération. L'étude à la caméra infra-rouge a été réalisée avec le concours de P. Privet. J. Cazaubieilh a fourni les micrographies acoustiques. Au Conservatoire National des Arts-et-Métiers, A. Kerboua a mis au point le système de caractérisation par imagerie de réflexion, en collaboration avec D. Duval et B. Rougié. 


\section{Bibliographie}

[1] Sнimbo M. et al., J. Appl. Phys. 60 (1986) 2987-2989.

[2] FurukAwA K. et al., Ext. abstr., 18th (1986 International) Conference on Solid State Devices and Materials, Tokyo (1986) pp. 533-536.

[3] Black R. D. et al., J. Appl. Phys. 63 (1988) 27732777.

[4] Nakagawa A. et al., IEEE IEDM Tech. Digest (1986) pp. 122-125.

[5] Goodenough F., Electronic Design (July 13, 1989) 31.

[6] Haisma J., J. Phys. Colloq. France 49 (1988) C4-3-8.

[7] Tenerz L. et HöK B., Electron. Lett. 22 (1986) 6162.

[8] Dessertenne B. et Dieumgard D., Proc. Europ. SOI workshop, Meylan, France (03.1988).

[9] GOTOU H., J. Phys. Colloq. France 49 (1988) C4471-480.

[10] Bengtsson B. et Engström O., J. Phys. Colloq. France 49 (1988) C4-63-66.

[11] Bengtsson B. et Engström O., J. Appl. Phys. 66 (1989) 1231-1239.

[12] Li Hui et al., Appl. Surf. Sci. 30 (1987) 397-401.

[13] Wallis G. et Pomerantz D. I., J. Appl. Phys. 40 (1969) 3946-3949.

[14] Anthony T. R, J. Appl. Phys. 58 (1985) 1240-1247.

[15] Gotou H. et al., Fujitsu Sci. Tech. J. 24 (1988) 408417.
[16] Chen Yi et Zhong Xiang-Fu, Proc. ICMPC (1988) pp. 195-198.

[17] UCHIDA Y. et al., Jpn. J. Appl. Phys. 11 (1986) 16331639.

[18] Lowen W. K., Broge E. C., J. Phys. Chem. 65 (1961) 16-19.

[19] Glang R. et Gregor L. V., Handbook of thin film technology, ed. L. I. Maissel et R. Glang (McGraw-Hill, N. Y.) 1970, pp. 7-45.

[20] Maugis D., Proc. $3^{\text {e }}$ Coll. Inter. Phys. Chim. Surfaces, Le Vide 186 (1977) 1-19.

[21] Lasky J. B., Appl. Phys. Lett. 48 (1986) 78-80.

[22] Latimer W. M., Rodebush W. H., J. Am. Chem. Soc. 42 (1920) 1419-1433.

[23] Marechal Y., La Recherche 20 (1989) 482-489.

[24] Maszara W. P., Goetz G., Caviglia A. et McKitTERICK J. B., J. Appl. Phys. 64 (1988) 49434950.

[25] ILeR R. K., The Chemistry of Silica (J. Wiley \& Sons, N. Y.) 1979, p. 625.

[26] Furukawa K., Nakagawa A., Appl. Surf. Sci. 41/42 (1989) 627-632.

[27] Stengl R., Ahn K.-Y. et Gösele U., Jpn. J. Appl. Phys. 27 (1988) L 2364-L 2366.

[28] HarendT C., Proc. Transducer's, Montreux (juin 1989). 